\title{
Los Programas de Ingeniería en Sistemas de Información y Control de Gestión en Chile: Un Análisis Cuantitativo
}

\author{
Patricio E. Ramírez, Paul S. Leger y Carolina A. Fuentes \\ Universidad Católica del Norte, Escuela de Ciencias Empresariales, Larrondo 1281, Coquimbo-Chile. \\ (e-mail: patricio.ramirez@ucn.cl; pleger@ucn.cl; cfuentes@ucn.cl)
}

Recibido Nov. 7, 2013; Aceptado Dic. 31, 2013; Versión final recibida Feb. 1, 2014

\begin{abstract}
Resumen
El objetivo principal de este trabajo es analizar cuantitativamente los contenidos de programas de Ingeniería en Sistemas de Información y Control de Gestión en Chile. El método utilizado se basa en la recolección y clasificación de los contenidos incorporados en las mallas de los programas. Los datos obtenidos se analizan con la técnica estadística Kruskal-Wallis. Los resultados indican que existen diferencias significativas entre estos nuevos programas y las ingenierías tradicionales. Además el estudio incorpora un análisis en relación a programas similares en el extranjero y discute otros aspectos sobre las instituciones que forman estos profesionales. Este estudio es el primero sobre este tipo de programas de formación superior en Chile.
\end{abstract}

Palabras clave: educación de la ingeniería, currículo, sistemas de información, control de gestión

\section{Engineering in Management Information Systems and Management Control Programs in Chile: A Quantitative Analysis}

\begin{abstract}
The main goal of this paper is to quantitatively analyze the contents of engineering in management information systems and management control programs in Chile. The methodology used is based on the collection and classification of the contents of these programs. The data obtained were analyzed with a statistical technique known as Kruskal-Wallis. The results show that there are significant differences between the new and the traditional engineering programs. In addition, this study involves an analysis that compares the similarity between Chilean and foreign programs and discusses other aspects of the institutions that form these professionals. This paper presents a pioneer study about these university programs in Chile.
\end{abstract}

Keywords: engineering education, curriculum, information systems, management control 


\section{INTRODUCCIÓN}

El estudio de los perfiles profesionales y del diseño curricular son temas relevantes para la investigación en educación superior, ejemplos de estos estudios pueden ser encontrados en Carpes y Magni (2009) y Avagliano y Vega (2013). Dentro de esta área temática, el presente trabajo entrega un analisis cuantitativo orientado a apoyar la definición de perfiles profesionales y el diseño curricular de un nuevo tipo de ingeniero empresarial en Chile.

La diferencia fundamental entre la gestión empresarial de hace algunas décadas y la del presente reside en el nivel de complejidad (Suárez-Tirado, 2013): cada vez más, la vida empresarial ofrece lo impredecible, lo sorprendente y lo inesperado. Este nuevo escenario de aumento en esta complejidad desafía a los gestores en cómo medir el pasado, controlar el presente y proyectar el futuro. Una respuesta a este desafío se ha asociado a la ciencia de datos o Big Data (Philip et al., 2013; Manyika et al., 2011; Sargut y McGrath, 2011, Albarrán y Salgado, 2013). Además, producto del enorme y continuo crecimiento en las capacidades de almacenamiento, transmisión y procesamiento de datos, el interés asociado a la ciencia de datos ha aumentado en forma exponencial.

Desde una perspectiva histórica, en la década de los noventa la "inteligencia de negocios" se convirtió en un vocablo popular para describir esta actividad. Al comienzo de este siglo la expresión "analítica en la empresa" se introdujo para representar este componente clave de análisis (Davenport y Harris, 2007). Y más recientemente, el término Big Data ha sido utilizado para describir los conjuntos de datos y técnicas de análisis en aplicaciones que son tan grandes y complejas que requieren avanzadas tecnologías de almacenamiento, gestión, análisis y visualización de datos (Chen et al., 2012). Es así que cada día más y más empresas necesitan profesionales que puedan traducir las enormes cantidades de datos existentes en valor para el negocio, de hecho, el científico de datos se ha propuesto como uno de los trabajos más atractivos para la próxima década (Davenport y Patil, 2012). Desafortunadamente, a pesar de esta creciente oportunidad, la demanda global de estos profesionales supera ampliamente la oferta de estas habilidades analíticas (Manyika et al., 2011; Kelly et al., 2013).

En el contexto anterior, en diversos países han surgido iniciativas de formación para responder a esta demanda. Estos programas integran estadísticas, ciencias de la computación, sistemas de información y gestión (ejemplos: MSc in Business Analytics, New York University; MSc in Data Science, Auckland University; MSc in Data Science, Dundee University). De igual forma, varias universidades chilenas han comenzado a ofertar un nuevo programa de ingeniería cuyo perfil se asocia a responder a las necesidades empresariales referidas anteriormente. Si bien el nombre de estos programas tiene un grado de variación entre estas instituciones, se puede denominar como Ingeniería en Sistemas de Información y Control de Gestión. Naturalmente y dado lo reciente de este nuevo perfil de ingeniero, el mercado laboral desconoce su formación. Además, existe una confusión entre los estudiantes al momento de seleccionar entre estos programas de estudios y otros de ingeniería tradicional, pues a primera vista son similares, y algo más preocupante aún, la comunidad universitaria en general no posee claridad sobre este nuevo campo de estudio. Estos hechos generan al menos tres problemas: el mercado laboral contrata a los egresados de estas carreras para cargos disfuncionales; existe una deserción de aquellos estudiantes que no entendían al momento de ingresar que áreas se estudiaban en el programa; y se dificulta la elección de docentes y la asignación de recursos para estos programas.

Con el propósito de dar luz sobre este nuevo perfil de ingeniero, este trabajo explora la oferta de programas existentes en Chile y los compara con los programas de ingeniería tradicional cercanos al perfil: Ingeniería Civil en Computación, Ingeniería Civil Industrial e Ingeniería Comercial. La contribución del estudio es distinguir explícitamente en que disciplinas se pone énfasis en estos nuevos programas, posibilitando con ello el contraste con las demandas de profesionales orientados a la ciencia de datos.

El desarrollo de este trabajo se ha estructurado como sigue. Primero se presenta la metodología utilizada, luego se entregan los resultados y su discusión, y finalmente se expone un grupo de conclusiones.

\section{METODOLOGÍA}

Para explorar y comparar el programa de estudio de Ingeniería en Sistemas de Información y Control de Gestión este trabajo se basa en la metodología propuesta por Reichgelt et al. (2004). La elección de esta metodología se basa en su reiterada aplicación en trabajos académicos anteriores (Aasheim et al.,2006; Abernethy et al., 2005; Tedre et al., 2009). Esta metodología usa la malla curricular de la carrera a estudiar y de otras carreras similares dentro de un conjunto de universidades. Cada asignatura de las carreras es clasificada en una disciplina de estudio (ej. computación, administración, economía, etc.). Luego, se explora la malla curricular y se compara con otras de acuerdo al nivel de presencia de las disciplinas de estudios. A continuación, se explica cómo está metodología fue aplicada en nuestro trabajo. 
Programas y Universidades Seleccionadas. Este trabajo considera el programa de pregrado Ingeniería en Sistemas de Información y Control de Gestión (INGESIS) o similares en nombre, es decir, programas cuyos nombres calcen con términos como "Control de Gestión", "Sistemas de Información", "Sistemas empresarial". Para comparar este programa de estudios, se consideraron tres programas tradicionales de ingeniería ofrecidos en Chile: Ingeniería Comercial (INGECO), Ingeniería Civil en Computación (INGECOM) e Ingeniería Civil Industrial (ICl)

Con respecto a las instituciones universidades donde se dictan estos programas, se recogieron datos de todas las universidades cuyos programas adoptan el Sistema de Crédito académicos Transferibles (SCT) y ofrecen estos cuatro programas. SCT permite a los alumnos tener asignaturas intercámbiales entre universidades, por ejemplo, un curso (ej. "Programación 1"), de una universidad A es equivalente (en carga total de trabajo necesaria para completarlo) al mismo curso de una universidad B. Las universidades analizadas son:

\section{Universidad Andrés Bello \\ Universidad Arturo Prat \\ Universidad Austral de Chile \\ Universidad Católica del Norte \\ Universidad de Chile \\ Universidad de Talca \\ Universidad de Tarapacá \\ Universidad Diego Portales}

Disciplinas de Estudios Seleccionadas. Cada asignatura de los programas fue clasificada en una disciplina de estudio. Para clasificar las disciplinas se usó Australian Standard Research Classification (ARC, 2013). Concretamente, este artículo clasifica cada asignatura en las siguientes disciplinas:

Ciencias de las Matemáticas

Ciencias de la Información, Computación y Servicios

Economía

Comercio, Administración, Turismo y Servicios

Ley y Justicia

Otras: Cualquier otra asignatura que no esté en las disciplinas anteriores

Recolección de datos y técnicas estadísticas. La recolección de datos se realizó en enero de 2013. Mayoritariamente los datos de los programas y asignaturas fueron tomados de los sitios Web oficiales de las instituciones. Solo en los casos donde no fue posible obtener estos datos directamente de la Web, ellos fueron solicitados por correo electrónico al encargado del programa en esa institución. En total se recolectaron datos de 32 programas. Estos datos se registraron en SPSS 15.0. Con el fin de analizar los resultados se usó la prueba de Kruskal-Wallis (Kruskal y Wallis, 1952), que es una alternativa no paramétrica a ANOVA.

\section{ANALISIS DE DATOS Y RESULTADOS}

Para cada programa de estudio seleccionado, se clasifican sus asignaturas en una disciplina de estudio. Este proceso fue repetido para cada universidad seleccionada. Las tablas 1 a 4 presentan los resultados para INGESIS, INGECO, ICI y INGECOM, respectivamente. En la tabla 5, se resumen el número promedio de cursos por disciplina.

Tabla 1: Número de asignaturas de INGESIS por disciplina.

\begin{tabular}{|l|c|c|c|c|c|}
\hline & \multicolumn{5}{|c|}{ Disciplina } \\
\hline Institución & Matemáticas & Computación & Economía & Administración & Derecho \\
\hline U. Andrés Bello & 3 & 16 & 0 & 4 & 0 \\
\hline U. Arturo Prat & 6 & 10 & 0 & 28 & 3 \\
\hline U. Austral de Chile & 5 & 8 & 5 & 24 & 1 \\
\hline U. Católica del Norte & 6 & 7 & 4 & 15 & 3 \\
\hline U. de Chile & 6 & 5 & 4 & 24 & 1 \\
\hline U. de Talca & 3 & 12 & 2 & 17 & 0 \\
\hline U. de Tarapacá & 4 & 7 & 3 & 26 & 4 \\
\hline U. Diego Portales & 5 & 5 & 3 & 24 & 4 \\
\hline Promedio $(n=8)$ & 4,75 & 8,75 & 2,63 & 20,25 & 2,00 \\
\hline
\end{tabular}


Tabla 2: Número de asignaturas de INGECO por disciplina.

\begin{tabular}{|l|c|c|c|c|c|}
\hline & \multicolumn{5}{|c|}{ Disciplina } \\
\hline Institución & Matemáticas & Computación & Economía & Administración & Derecho \\
\hline U. Andrés Bello & 5 & 2 & 5 & 26 & 1 \\
\hline U. Arturo Prat & 6 & 3 & 5 & 26 & 3 \\
\hline U. Austral de Chile & 6 & 3 & 8 & 29 & 0 \\
\hline U. Católica del Norte & 5 & 1 & 7 & 21 & 2 \\
\hline U. de Chile & 6 & 1 & 14 & 27 & 1 \\
\hline U. de Talca & 8 & 2 & 9 & 27 & 0 \\
\hline U. de Tarapacá & 6 & 2 & 7 & 22 & 3 \\
\hline U. Diego Portales & 6 & 0 & 9 & 19 & 3 \\
\hline Promedio (n=8) & 6,00 & 1,75 & 8,00 & 24,63 & 1,63 \\
\hline
\end{tabular}

Tabla 3: Número de asignaturas de ICI por disciplina.

\begin{tabular}{|l|c|c|c|c|c|}
\hline & \multicolumn{5}{|c|}{ Disciplina } \\
\hline Institución & Matemáticas & Computación & Economía & Administración & Derecho \\
\hline U. Andrés Bello & 7 & 4 & 1 & 12 & 0 \\
\hline U. Arturo Prat & 8 & 4 & 2 & 9 & 0 \\
\hline U. Austral de Chile & 9 & 7 & 2 & 14 & 0 \\
\hline U. Católica del Norte & 8 & 6 & 2 & 6 & 0 \\
\hline U. de Chile & 11 & 3 & 3 & 8 & 0 \\
\hline U. de Talca & 9 & 3 & 1 & 14 & 0 \\
\hline U. de Tarapacá & 7 & 6 & 2 & 12 & 1 \\
\hline U. Diego Portales & 8 & 3 & 3 & 7 & 1 \\
\hline Promedio $(\mathrm{n}=8)$ & 8,38 & 4,50 & 2,00 & 10,25 & 0,25 \\
\hline
\end{tabular}

Tabla 4: Número de asignaturas de INGECOM por disciplina.

\begin{tabular}{|l|c|c|c|c|c|}
\hline & \multicolumn{5}{|c|}{ Disciplina } \\
\hline Institución & Matemáticas & Computación & Economía & Administración & Derecho \\
\hline U. Andrés Bello & 5 & 20 & 0 & 2 & 0 \\
\hline U. Arturo Prat & 8 & 23 & 1 & 1 & 0 \\
\hline U. Austral de Chile & 8 & 32 & 1 & 3 & 0 \\
\hline U. Católica del Norte & 8 & 19 & 1 & 0 & 0 \\
\hline U. de Chile & 9 & 17 & 1 & 2 & 0 \\
\hline U. de Talca & 6 & 29 & 1 & 3 & 0 \\
\hline U. de Tarapacá & 8 & 28 & 1 & 2 & 1 \\
\hline U. Diego Portales & 8 & 13 & 1 & 1,88 & 0,13 \\
\hline Promedio $(\mathrm{n}=8)$ & 7,50 & 22,63 & 0,88 & & 0 \\
\hline
\end{tabular}

Tabla 5: Número promedio de asignaturas por programa analizado según disciplina.

\begin{tabular}{|l|c|c|c|c|c|}
\hline & \multicolumn{5}{|c|}{ Disciplina } \\
\hline Programa & Matemáticas & Computación & Economía & Administración & Derecho \\
\hline INGESIS & 4,75 & 8,75 & 2,63 & 20,25 & 2,00 \\
\hline INGECO & 6,00 & 1,75 & 8,00 & 24,63 & 1,63 \\
\hline ICI & 8,38 & 4,50 & 2,00 & 10,25 & 0,25 \\
\hline INGECOM & 7,50 & 22,63 & 0,88 & 1,88 & 0,13 \\
\hline
\end{tabular}

Tabla 6: Prueba de Kruskal-Wallis, indica diferencia en los contenidos disciplinarios de los programas analizados. a. Prueba de Kruskal-Wallis; b. Variable de agrupación: Programa

\begin{tabular}{|l|c|c|c|c|c|}
\hline & \multicolumn{5}{|c|}{ Rango promedio por disciplina } \\
\hline Programa & Matemáticas & Computación & Economía & Administración & Derecho \\
\hline INGESIS & 7,13 & 19,75 & 16,00 & 22,00 & 22,38 \\
\hline INGECO & 12,63 & 4,88 & 28,38 & 26,00 & 21,13 \\
\hline ICI & 25,13 & 13,00 & 14,38 & 13,50 & 12,00 \\
\hline INGECOM & 21,13 & 28,38 & 7,25 & 4,50 & 10,50 \\
\hline \multicolumn{7}{|c|}{ Estadísticos de contraste $(\mathrm{a}, \mathrm{b})$} \\
\hline Chi-cuadrado & 18,988 & 27,339 & 21,671 & 24,964 & 12,191 \\
\hline Grados de libertad & 3 & 3 & 3 & 3 & 3 \\
\hline Significación asintótica & 0,0003 & 0,0000 & 0,0001 & 0,0000 & 0,0068 \\
\hline
\end{tabular}


Con el propósito de revisar si existen diferencias significativas entre el número de cursos por disciplina entre los programas estudiados se realizaron pruebas Kruskal-Wallis, las cuales permiten probar si un grupo de datos proviene de la misma población. Esta técnica no requiere normalidad de los datos. En específico, Kruskal-Wallis se basa en ordenar las observaciones en rangos, para luego calcular el rango promedio por subgrupo de análisis. Estos últimos son utilizados para determinar si estos subgrupos son de la misma población, en ese caso, el valor $p>0.05$. En caso contrario $(p<0.05)$, los datos proviene de distintas fuentes. La tabla 6 resume los resultados de estas pruebas. A continuación, se comenta estos resultados por disciplina.

Matemáticas. La comparación de los rangos promedio muestra que el número de asignaturas de matemáticas los programas de $\mathrm{ICI}(25,13)$ superan significativamente a los programas de INGESIS $(7,13)$. El rango promedio de ICI es seguido en orden descendente por INGECOM $(21,13)$ y luego por INGECO $(12,63)$. La prueba estadística de Kruskal-Wallis indica que la diferencia entre los programas en relación con la disciplina de matemáticas es significativa $(p<0,001)$. Este resultado indica una clara carencia de los programas de INGESIS en orden de entregar habilidades para aplicar técnicas de análisis numérico complejos para apoyar la generación de valor empresarial.

Computación. La comparación de los rangos promedio muestra que el número de asignaturas de computación los programas de INGECOM $(28,38)$ superan significativamente a los otros programas. EI rango promedio de INGECOM es seguido en orden descendente por INGESIS $(19,75)$, ICI $(13,00)$ e INGECO $(4,88)$. La prueba estadística de Kruskal-Wallis indica que la diferencia entre los programas en relación con la disciplina de computación es significativa $(p<0,001)$. Este resultado nos señala la clara cercanía en la disciplina de computación entre los programas de INGESIS y los de INGECOM, así como la importante distancia en este ámbito entre INGESIS e INGECO. Por otra parte, este hallazgo revela el potencial desarrollo de habilidades para el uso de tecnologías de almacenamiento, gestión, análisis y visualización de datos en los programas de INGESIS.

Economía. La comparación de los rangos promedio muestra que el número de asignaturas de economía los programas de INGECO $(28,38)$ superan significativamente a los otros programas. El rango promedio de INGECO es seguido en orden descendente por INGESIS $(16,00)$, ICI $(14,38)$ e INGECOM $(7,25)$. La prueba estadística de Kruskal-Wallis indica que la diferencia entre los programas en relación con la disciplina de economía es significativa $(p<0,001)$. Este resultado es consistente con un perfil profesional de INGESIS que capacite para entender los fenómenos económicos globales y como ellos afectan a la realidad empresarial.

Administración. La comparación de los rangos promedio muestra que el número de asignaturas de administración los programas de INGECO $(26,00)$ superan significativamente a los otros programas. EI rango promedio de INGECO es seguido en orden descendente por INGESIS $(22,00)$, ICI $(13,50)$ e INGECOM (4,50). La prueba estadística de Kruskal-Wallis indica que la diferencia entre los programas en relación con la disciplina de administración es significativa $(p<0,001)$. Este resultado indica la clara identidad del perfil de este nuevo ingeniero, esto es, un importante dominio de la disciplina de administración para traducir los datos en valor para el negocio (Davenport y Patil, 2012).

Derecho. La comparación de los rangos promedio muestra que en número de asignaturas de derecho los programas de INGESIS $(22,38)$ superan significativamente a los otros programas. El rango promedio de INGESIS es seguido en orden descendente por INGECO $(21,13)$, ICI $(12,00)$ e INGECOM $(10,50)$. La prueba estadística de Kruskal-Wallis indica que la diferencia entre los programas en relación con la disciplina de derecho es significativa $(p<0,01)$. Este resultado ratifica la importancia de entender el entorno empresarial en el perfil de los profesionales INGESIS, y en particular, como los aspectos legales afectan el que hacer empresarial, restringiendo o habilitando la toma de decisiones.

Basados en la tabla 5, es posible construir la figura 1. Este resultado muestra el nivel de presencia de las disciplinas en los cuatro programas de pregrado analizados. Por ejemplo, la presencia de la disciplina de la computación en INGECOM es sumamente alta, así mismo, la presencia de la disciplina de administración en INGECO.

Para realizar un análisis más refinado y basados en la tabla 6, se construye la figura 2. Esta figura muestra las diferencias entre los programas, según sus rangos promedios entre el número de asignaturas en cada disciplina. Este resultado resume las diferencias entre el los nuevos programas de INGESIS y los otros programas de ingeniería tradicional analizados. En la figura 2, INGESIS es claramente un perfil diferenciado de las los otros programas, y si bien se acerca en algunas disciplinas a alguno de ellos, en su conjunto es único. En este perfil, se puede describir como un profesional con una mirada global (economía y derecho) de la empresa (administración) y las tecnologías de información (computación). 


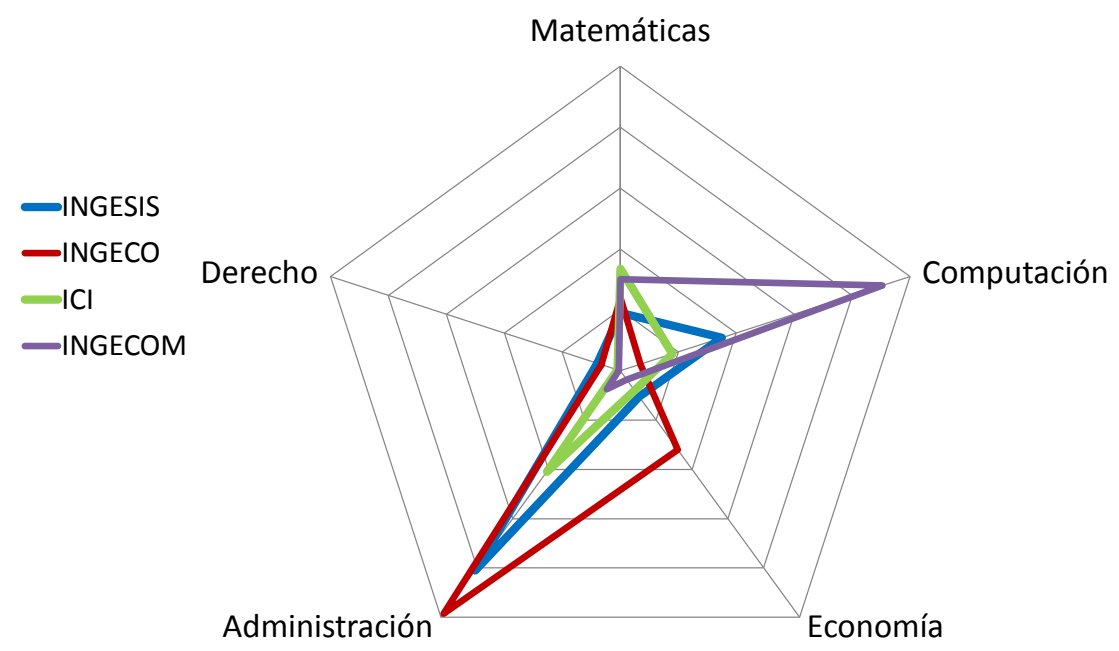

Fig. 1: Representación gráfica del número de asignaturas promedio por disciplina de los programas analizados.

Por último, deseamos comparar los resultados obtenidos en el caso de INGESIS con otros estudios en el contexto internacional. Considerando el currículo de exitosos programas equivalentes a INGESIS en Estados Unidos, Europa y Asia, La Paz et al. (2010) indican que las tres áreas con mayor número de asignaturas son implantación de tecnologías de información, administración, y computación, en ese orden. Los resultados de nuestro estudio indican que existe congruencia de esos hallazgos con los programas nacionales en la segunda y tercera áreas, sin embargo no hay evidencias que la implantación de tecnologías de información esté considerada en forma importante en los programas de INGESIS en Chile, lo que indica una clara incongruencia. Por otra parte, McGee y Preobragenskaya (2009) indican que en programas asociados al control de gestión en Estados Unidos y en Rusia, las asignaturas de las áreas de administración más economía suman el 70,5\% y 76,2\% en estos países respectivamente. Estos hallazgos son muy cercanos a los resultados asociados a INGESIS en Chile.

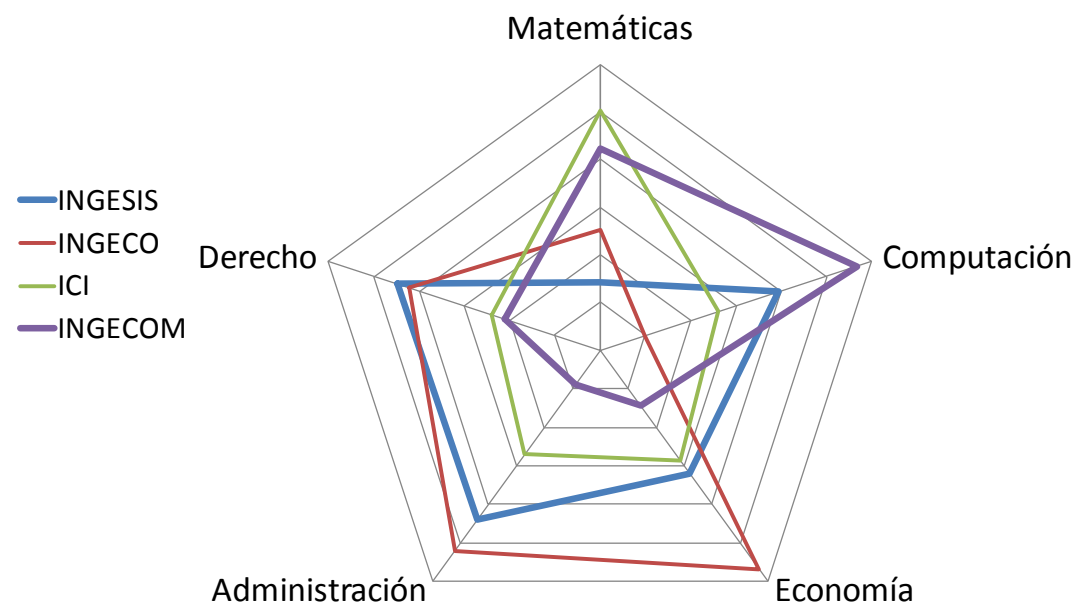

Fig. 2: Representación gráfica de los rangos promedios de asignaturas por disciplina de los programas analizados.

\section{CONCLUSIONES}

En este trabajo se exploró la oferta de los programas de Ingeniería en Sistemas de Información y Control de Gestión en Chile y los comparó con programas de ingeniería tradicional. Para efectuar este análisis se utilizó una aproximación metodológica cuantitativa. Basados en la revisión de treinta y dos programas de estudio se pudieron extraer elementos claves para la discusión. 
Los resultados del estudio indican diferencias entre la formación del profesional de Ingeniería en Sistemas de Información y Control de Gestión y la formación de otras ingenierías tradicionales. Si bien la formación promedio del Ingeniero en Sistemas de Información y Control de Gestión entrega las habilidades necesarias para apoyar a las organizaciones en las tareas asociadas a Big Data, existe una carencia en el área de matemáticas en comparación con ingenierías tradicionales.

Algunas recomendaciones que se pueden extraer del análisis realizado para los programas de Ingeniería de Sistemas de Información y Control de Gestión en Chile. Primero, dado que la formación del Ingeniero de Sistemas de Información y Control de Gestión en Chile contempla un conjunto de contenidos disciplinarios que lo distinguen de otras ingenierías tradicionales, es necesario que las instituciones que imparten estos programas difundan con mayor fuerza y precisión el aporte de este nuevo tipo de profesionales a la sociedad. Segundo, se destaca en el análisis de resultados la carencia de contenidos disciplinarios en implantación de tecnologías de información (tales como estándares de gestión de proyectos, modelos aceptación individual de tecnologías, y gestión del cambio). En consecuencia, es necesario que las instituciones que dictan estos programas discutan en los futuros rediseños como integrar esos contenidos. $Y$ finalmente, considerando la relativa coincidencia en los actuales diseños y las oportunidades de mejoras futuras de estos programas, sería de gran utilidad crear una red de universidades que de un soporte a los procesos de acreditación y rediseño de programas a nivel nacional.

Entre las limitaciones de este estudio está no considerar los cursos optativos en los programas analizados. Sin embargo, desde que estos cursos son de diversas índole pero están normalmente dentro del área de desarrollo de competencias profesionales (ejemplos: gestión de proyectos, sistemas empresariales, control en organizaciones públicas), creemos que esta limitación no afecta en forma substancial los resultados.

\section{REFERENCIAS}

Aasheim, C., Lee, C. K. y Reichgelt, H. An implementation of the IT fundamentals knowledge area in an introductory IT course. Journal of Information Technology Education, 5, 373-388 (2006).

Abernethy, K., Gabbert, P., Treu, K., Piegari, G. y Reichgelt, H. Impact of the emerging discipline of information technology on computing curricula: some experiences. Journal of Computing Sciences in Colleges, 21 (2), 237-243 (2005).

Albarrán, S. y Salgado, M. La Inteligencia Analítica y la Competitividad en las Empresas. RECAI Revista de Estudios en Contaduría, Administración e Informática, 24-47 (2013).

ARC, Research Fields, Courses and Disciplines (RFCD) classification (en línea), 1998. Australian Research Council, http://www.arc.gov.au. Acceso: 25 de Marzo, (2013).

Avagliano, A. R. y Vega, S. A. Mejora del Proceso de Enseñanza y Aprendizaje en la Carrera de Ingeniería de Ejecución Mecánica: Diseño Micro-curricular Basado en Resultados de Aprendizaje. Formación Universitaria, 6 (4), 3-12 (2013).

Carpes, A. D. y Magni, J. A. Búsqueda de un Nuevo Perfil para el Farmacéutico: la Readaptación de los Currículos en Cursos de Farmacia. Formación Universitaria, 2 (3), 3-10 (2009).

Chen, H., Chiang, R. H. L., y Storey, V. C. Business intelligence and analytics: from big data to big impact. MIS Quarterly, 36 (4), 1165-1188 (2012).

Davenport, T. H. y Harris, J. G. Competing on analytics: the new science of winning. Boston: Harvard Business School Press, (2007).

Davenport, T. H. y Patil, D. J. Data scientist: the sexiest job of the 21st century. Harvard Business Review, 90 (10), 70-76 (2012).

Kelly, K., Marlino, M., Mayernik, M. S., Allard, S., Tenopir, C., Palmer, C. L., y Varvel Jr, V. E. Model Development for Scientific Data Curation Education. International Journal of Digital Curation, 8 (1), 255-264, (2013).

Kruskal, W.H. y Wallis, W.A. Use of ranks in one-criterion variance analysis. Journal of the American Statistical Association, 47 (260), 583-621 (1952). 
La Paz, A., Laengle, S. y Cancino C. The core skills of the CIO we need. DYNA, 163, 172-180, (2010).

Manyika, J., Chui, M., Brown, B., Bughin, J., Dobbs, R., Roxburgh, C. y Byers, A. H. Big data: The next frontier for innovation, competition, and productivity (en línea), 2011. McKinsey Global Institute, http://www.mckinsey.com. Acceso: 15 de Enero, (2013).

McGee, R. W. y G.G. Preobragenskaya. Accounting education in Russia and the USA: A comparative study. Accounting Reform in Transition and Developing Economies, 317-335 (2009).

Philip, T. M., Schuler-Brown, S. y Way, W. A Framework for Learning About Big Data with Mobile Technologies for Democratic Participation: Possibilities, Limitations, and Unanticipated Obstacles. Technology, Knowledge and Learning, 18 (3), 1-18 (2013).

Reichgelt, H., Lunt, B., Ashford, T., Phelps, A., Slazinski, E. y Willis, C. A Comparison of Baccalaureate Programs in Information Technology with Baccalaureate Programs in Computer Science and Information Systems. Journal of Information Technology Education: Research, 3 (1), 19-24 (2004).

Sargut, G. y McGrath, R. G. Learning to live with complexity. Harvard Business Review, 89 (9), 68-76 (2011).

Suárez-Tirado, J. Control de gestión en la cadena de valor y los aportes de la contabilidad de gestión: estudio de caso de una compañía colombiana. Cuadernos de Contabilidad, 14 (34), 245-261 (2013).

Tedre, M., Bangu, N. y Nyagava, S. Contextualized IT education in Tanzania: Beyond standard IT curricula. Journal of Information Technology Education: Research, 8 (1), 101-124 (2009). 